\title{
THE DISTRIBUTION AND ECOLOGICAL PREFERENCES OF STONE CRAYFISH AUSTROPOTAMOBIUS TORRENTIUM (SCHRANK, 1803) (DECAPODA: ASTACIDAE) IN THE NORTH- WEST ROMANIA
}

\section{LUCIAN PÂRVULESCU}

\begin{abstract}
This paper presents data on geographical distribution and ecological preferences of Austropotamobius torrentium species following investigations of 104 sampling sites in the west and north-western Romania. Specimens were caught by actively searching the river bed, after which they were released. A detailed map of the distribution was prepared: the species was found to be living in 23 sampling sites from three basins: Crişul Alb, Crişul Negru and Crişul Repede. The current distribution of the species extends to the central and eastern part of the Codru Moma Mountains, western Bihor Mountains, eastern Pădurea Craiului Mountains, central and northern areas of the Vlădeasa Mountains and southern and central areas of the Plopiş Mountains. The northernmost population of Romania is found in the Valea Mare River, on the Plopiş Mountains. Generally, the species occurs on a substrate consisting primarily of stone and gravel. The species' preferred combination of riparian vegetation is Alnus sp. and Salix sp. in deciduous forests. An important number of investigated rivers, totalling 37, are occupied by the Astacus astacus species, data proving that there are no mixed populations. No other non-indigenous crayfish species were found during this study.
\end{abstract}

Résumé. Ce travail présente des données sur la distribution géographique et les préférences écologiques d'espèce Austropotamobius torrentium suite aux investigations sur 104 sites d'échantillonnage dans l'ouest et au nord-ouest de la Roumanie. Les spécimens ont été capturés par les recherchent activement au fond de la rivière, après ils ont été libérés. Une carte détaillée de la distribution a été préparé: l'espèce a été trouvée dans 23 rivières de trois bassins: Crișul Alb, Crișul Negru et Crișul Repede. La répartition actuelle de l'espèce s'étend à la partie centrale et orientale des Montagnes Codru Moma, dans l'ouest des Montagnes Bihor, la partie orientale des Montagnes Pădurea Craiului, la zone centre et du nord de la Montagne Vlădeasa et le centre et la zone du sud des Montagnes Plopiş. L'espèce se trouve généralement dans les rivières avec un substrat constitué principalement de pierres de taille variable et de gravier. La combinaison de la végétation riveraine préférée par l'espèce étudiée est représenté par: Alnus sp. et Salix sp., dans les forêts à feuilles caduques. La plus nordique population d'espèce $A$. torrentium de la Roumanie se trouve dans la rivière Valea Mare, dans les Montagnes Plopiş. Dans un nombre important de rivières étudiées, totalisant 37, a été trouvée l'espèce Astacus astacus. Ces données montrent qu'il n'y a pas des populations mixtes. Dans cette étude, aucune des espèces envahissantes n'a pas été trouvé dans les zones étudiées.

Key words: Astacus astacus, Austropotamobius torrentium, crayfish, distribution, ecology, endangered species, noble crayfish, stone crayfish.

\section{INTRODUCTION}

The stone crayfish Austropotamobius torrentium (Schrank, 1803) is one of the three indigenous crayfish species of the Romanian freshwater ecosystems, the others being the noble crayfish Astacus astacus (Linnaeus, 1758), and the narrow-clawed crayfish Astacus leptodactylus Eschscholtz, 1823 (Băcescu, 1967; Holdich et al., 2009; Pârvulescu, 2010). One non-native crayfish, the spiny-cheek crayfish Orconectes limosus (Rafinescque, 1817) (Pârvulescu et al., 2009), occurs in Romanian freshwater ecosystems. In the European Council's Directive 92/43 (assumed by Romanian law 57 / 2007), A. torrentium is rated as a "priority species", 
and therefore it is necessary to implement a series of management measures in the protected areas, among which the periodic measurement of population density is of great importance (Pârvulescu, 2007). Now, the actual IUCN status rates this species as "data deficient" (Füreder et al., 2010).

The European distribution of this species covers the central and southeastern areas of the continent, from Germany and the Czech Republic in the north, Luxembourg and France to the west, Greece to the south, and Romania, Bulgaria and Turkey in the east (Holdich et al., 2006). Austropotamobius torrentium usually lives in small and medium-sized rivers and streams (Vlach et al., 2009), requiring pristine waters (Svobodová et al., 2008; Pârvulescu et al., 2011). Over a period of decades, many factors could influence the geographical distribution of $A$. torrentium, for example, modification of its habitat and water pollution (Lowery \& Hogger, 1986; Gherardi and Holdich, 1999; Füreder et al., 2003; Lyons \& KellyQuinn, 2003), a lower rate of growth (Streissl \& Hödl, 2002) and lower fecundity (Maguire et al., 2010). Anthropogenic impacts such as organic pollution and river bed modification might have triggered the disappearance of the species from sections of the watercourse situated downstream of villages and towns (Pârvulescu et al., 2011). The high pressure caused by both invasive crayfish species and the crayfish plague pathogen (Aphanomyces astaci) has become a serious threat, which is almost unstoppable based on present knowledge. At the end of 2011, Orconectes limosus already occupies the first $105 \mathrm{~km}$ of the Romanian Danube, carrying the crayfish plague (Pârvulescu et al., 2012).

There are few data published regarding the freshwater crayfish species in Romania. The earliest records of A. torrentium in Romania place this species in the south-west of the country (Scriban, 1908). Entz (1912) also recorded the species in the south-west area, and additionally in the centre of the Transylvanian Plateau. Between 1935 and 1962 there were references to its presence in the south-west, west and north-west of Romania (Băcescu, 1967). The catalogue of the "Grigore Antipa" National Museum of Natural History in Bucharest lists the localities where the species was found, mainly based on Mihai Băcescu's collection (Petrescu \& Petrescu, 2010). These earlier observations are difficult to georeference because geographical coordinates were not given, and so it is hard to establish a clearly defined geographical area for the distribution of $A$. torrentium. Also, as these records are over 30 years old, it is very possible that the actual situation may no longer be the same. This paper provides evidence for and completes the data relating to the distribution and ecology, in order to assist in efficient conservation management for this species.

\section{MATERIAL AND METHODS}

During 2010 and 2011, field sampling was carried out in order to establish the distribution of $A$. torrentium in the permanent waters in the upper sections of the main courses and tributaries from the mountain areas and sub-mountain areas of the west and north-west of Romania. The extent of the investigated area is from north of the Mureş River, covering 14 geographical units (i.e. mountains, plateaus, hills). Tributaries of six hydrographic basins were investigated: the Crişul Alb, Crişul Negru, Crişul Repede, Someş, Mureş and Tisa rivers. The sections for sampling sites were chosen at random, and we investigated an average of 15 mainstreams per geographical unit, with the number for each depending on its size. Each sampling site contained, on average, a stretch of river of approximately 200 
$\mathrm{m}$ in length. The crayfish were collected using direct hand sampling from the river bed, by searching shores galleries, spaces between rocks and within the roots. The relative abundance (catch per unit effort, CPUE) of crayfish was estimated as the number of crayfish caught per $100 \mathrm{~m}$ length of river stretch. Where no crayfish were found, the sampling site was recorded as one with "no crayfish", but this was only confirmed after investigating at least $300 \mathrm{~m}$ of a river. The captured crayfish specimens were released at exactly the same location at which they were caught. The riverbed morphology, shelter use and riparian vegetation were recorded when observations of the presence of crayfish were made. These indicators were defined in terms of the percentages of different types in each sector. The bottom types included boulders, stones, gravel, sand and mud, and the shelter use was defined accordingly to the type where each specimen was found.

A digital distribution map at 1:50,000 scale was prepared, based on the georeferenced field data. Using GlobalMapper software, we obtained a map of distribution points by marking where we found each of the crayfish species, as well as the sites where none were found. Then, using InkScape, we coloured the distribution points to make the areas visible. The most-probable distribution area was established by analysis of the distribution of the points, taking into consideration the biogeography of the species and the watershed area. Ecological parameters were studied with the weighted average method. The weights were represented by the relative abundance (CPUE).

\section{RESULTS AND DISCUSSION}

In 60 of 104 investigated sampling sites, crayfish were present (Fig. 1), 23 populated by the stone crayfish (Austropotamobius torrentium) and 37 by the noble crayfish (Astacus astacus). Table 1 contains data summaries for the ecological parameters measured in the locations where $A$. torrentium specimens were found. The

Data summary for several parameters of the habitat measured at the sampling sites where A. torrentium was found.

Intervals with the highest frequencies are shaded.

\begin{tabular}{|c|c|c|c|c|c|c|c|c|}
\hline \multicolumn{9}{|c|}{ River bottom mixture } \\
\hline Type & \multicolumn{2}{|c|}{ boulders } & stones & \multicolumn{2}{|c|}{ gravel } & sand & \multicolumn{2}{|r|}{ mud } \\
\hline Frequency $(\%)$ & \multicolumn{2}{|l|}{6.8} & 31.8 & \multicolumn{2}{|c|}{43.5} & 12.7 & \multicolumn{2}{|r|}{5.2} \\
\hline \multicolumn{9}{|c|}{ Shelter use } \\
\hline Type & \multicolumn{2}{|c|}{ bank galleries } & \multicolumn{2}{|c|}{ under stones } & \multicolumn{2}{|c|}{ between roots } & \multicolumn{2}{|r|}{ free } \\
\hline Frequency $(\%)$ & \multicolumn{2}{|c|}{28.6} & \multicolumn{2}{|c|}{54.2} & \multicolumn{2}{|c|}{16.8} & \multicolumn{2}{|r|}{0.4} \\
\hline \multicolumn{9}{|c|}{ Tree coverage } \\
\hline Interval & \multicolumn{2}{|c|}{$0-20 \%$} & $21-40 \%$ & \multicolumn{2}{|c|}{$41-60 \%$} & \multicolumn{2}{|c|}{$61-80 \%$} & $81-100 \%$ \\
\hline Frequency $(\%)$ & \multicolumn{2}{|c|}{11.2} & 18 & \multicolumn{2}{|c|}{10.1} & \multicolumn{2}{|l|}{25.9} & 34.8 \\
\hline \multicolumn{9}{|c|}{ Riparian trees composition } \\
\hline Genus or type & 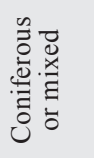 & 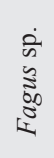 & 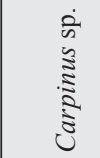 & 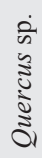 & 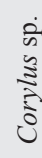 & 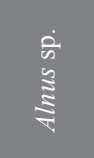 & $\begin{array}{l}\text { के } \\
\text { : } \\
\text { ¿ }\end{array}$ & $\frac{\bar{D}}{\overline{0}}$ \\
\hline Frequency $(\%)$ & 2.9 & 5.5 & 6.8 & 0.3 & 6.2 & 37.4 & 24.5 & 16.4 \\
\hline
\end{tabular}




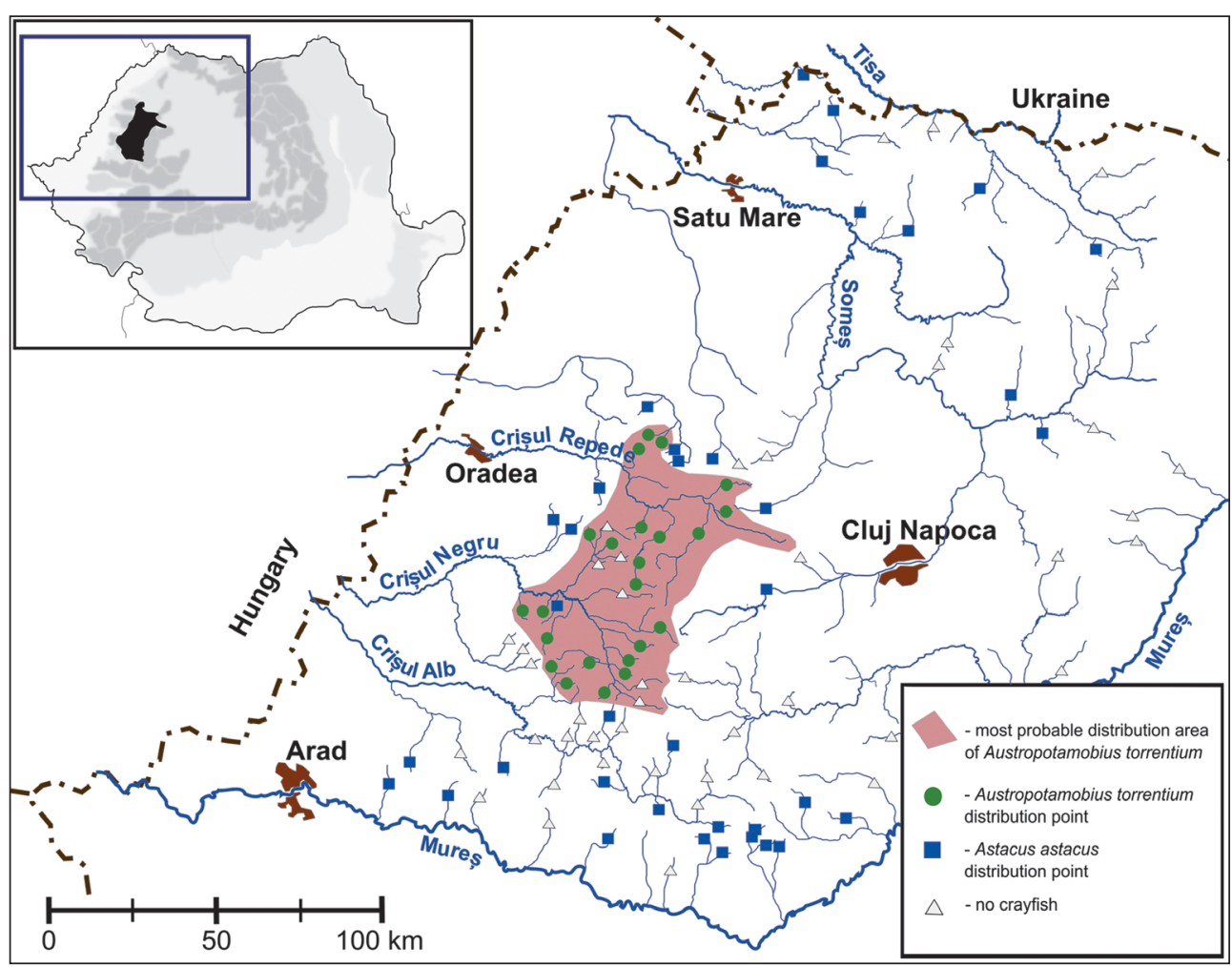

Fig. 1 - The distribution area of Austropotamobius torrentium and Astacus astacus obtained from data assembled during the 2010-2011 fieldwork in the west and north-west of Romania.

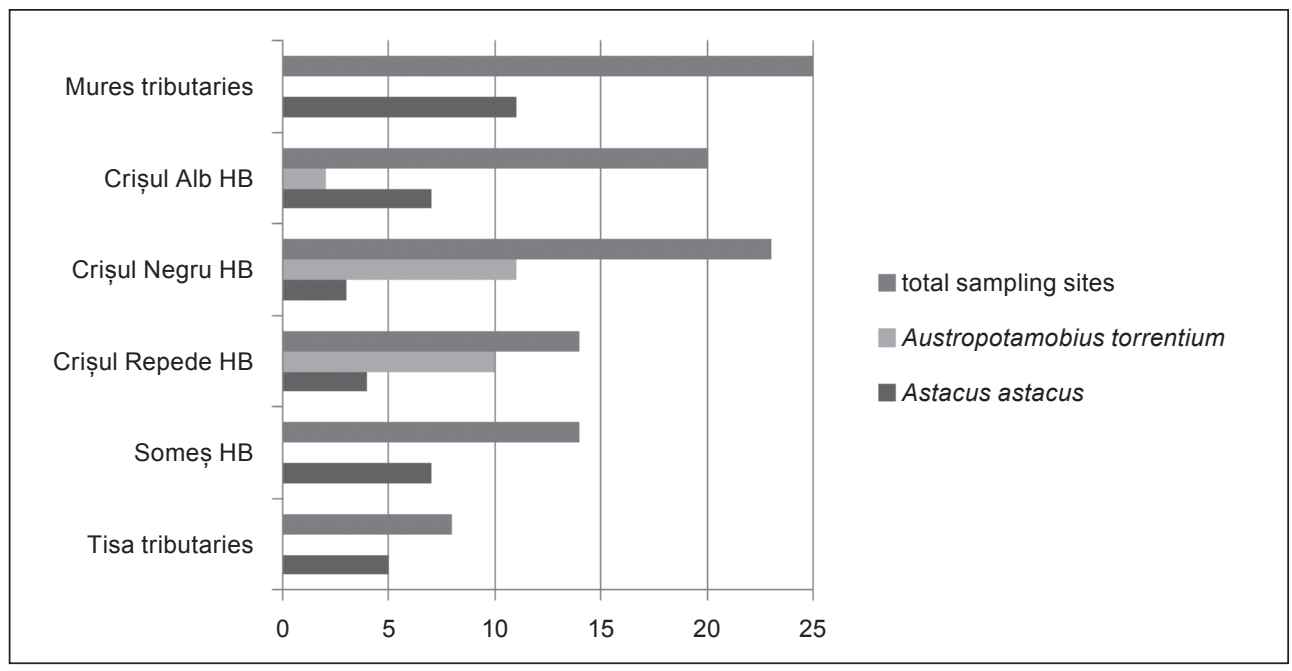

Fig. 2 - The frequency of crayfish species in the hydrographic basins, investigated during the 20102011 fieldwork in the west and north-west of Romania. 
species occurs on a substrate consisting primarily of stone and gravel, the preferred shelter is represented by the spaces under and between stones. The most frequent combination of riparian vegetation is Alnus sp. and Salix sp. These ecological results are similar to those presented in other publications (e.g. Machino \& Füreder, 2005).

The distribution results are following described according to the hydrographic basin from which they were obtained, from south to north.

The Mureş tributaries. Twenty-five sampling sites of the middle section of the Mureş River were investigated from the Trascău, Metaliferi and Zărandului mountains indicating the presence of only A. astacus crayfish species (Tab. 2).

The Crişul Alb hydrographic basin. Twenty sampling sites distributed in the area of the Codru Moma, Bihor, Zărand and Metaliferi mountains were investigated; A. torrentium was found in $10 \%$ of the investigated sites, A. astacus was identified in $35 \%$ of the investigated sites, and $55 \%$ did not harbour crayfish (Fig. 2, tab. 2). The highest altitude at which $A$. torrentium was found was at $370 \mathrm{~m}$, in the Valea Megheş River (Tab. 2).

The Crişul Negru hydrographic basin. Twenty-three sampling sites, situated in five geographical units, were investigated: the Vlădeasa, Plopiş, Pădurea Craiului, Bihor and Codru Moma mountains. Two crayfish species were identified: $A$. torrentium and A. astacus, the former being found in $47.8 \%$ of the investigated sites (Fig. 2, tab. 2). The location at the highest altitude was in the Boga Stream, at $560 \mathrm{~m}$, where we captured only one specimen. A. astacus was captured in three sampling sites (Tab. 2).

The Crişul Repede hydrographic basin. Fourteen sampling sites in several geographical units in this basin were investigated: the Meseş, Plopiş, Pădurea Craiului and Vlădeasa mountains. A. torrentium was found in $71.4 \%$ of the investigated sites (Fig. 2, tab. 2). The maximum altitude at which the species was found was $800 \mathrm{~m}$, in the Valea Ieduţului Stream. The highest population density registered for the species in the investigated area was 55.8 individuals per $100 \mathrm{~m}$ of river stretch on the Valea Mare River. A. astacus was captured in four sampling sites (Tab. 2).

The Someş hydrographic basin. Fourteen sampling sites were investigated in the area of the Gutâi, Țibleş, Meseş, and Gilău mountains and western part of the Transylvanian Plateau. Only A. astacus was found (Tab. 2).

The Tisa tributaries. Eight sampling sites were investigated, situated on the Oaş, Gutâi and Ţibleş mountains, only A. astacus being found (Tab. 2).

Compared to populations of $A$. torrentium from the south-west of Romania (Pârvulescu \& Petrescu, 2010), the western and north-western populations are scarcely represented. These populations occupy the central and eastern part of the Codru Moma Mountains, the western Bihor Mountains, the eastern Pădurea Craiului Mountains, the central and northern Vlădeasa Mountains and the southern and central Plopiş Mountains (Fig. 2). The majority of this area is included in the Apuseni Nature Park and several Natura 2000 sites. The northern population of this species in Romania is represented by the Valea Mare River, in the Plopiş Mountains. Contrary to information presented by the collection of "Grigore Antipa" National Museum of Natural History of Bucharest (Petrescu \& Petrescu, 2010), the species was not found in the Tisa tributaries, where only A. astacus was present.

The non-indigenous $O$. limosus does not represent a significant threat for A. torrentium in the west and north-west of Romania. It is known that this invasive species spreads through large rivers, and colonization of small tributaries is very rare, and only through human mediation (Petrusek et al., 2006; Pârvulescu et al., 2012). The pathway for $O$. limosus colonization in the future could be the Mureş and Tisa 
Table 2

Crayfish sampled rivers toponyms, geographic coordinates (Stereo 70), and captured specimens.

\begin{tabular}{|c|c|c|c|c|}
\hline $\begin{array}{c}\text { Rivers } \\
\text { toponyms }\end{array}$ & Geographic coordinates & 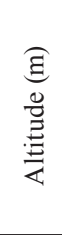 & 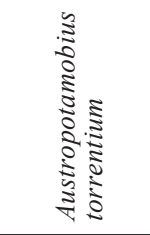 & 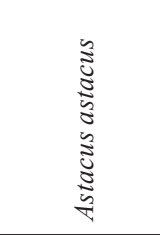 \\
\hline \multicolumn{5}{|c|}{ Mureş tributaries } \\
\hline Tămăşeşti & $46^{\circ} 01^{\prime} 32^{\prime \prime} \mathrm{N} / 22^{\circ} 30^{\prime} 39^{\prime \prime} \mathrm{E}$ & 340 & - & 1 d, 5 우우 \\
\hline Carpăn & $46^{\circ} 03^{\prime} 58^{\prime \prime} \mathrm{N} / 22^{\circ} 55^{\prime} 51^{\prime \prime} \mathrm{E}$ & 179 & - & $2 \hat{\jmath}, 1$ q \\
\hline Căianu & $46^{\circ} 02^{\prime} 00^{\prime \prime} \mathrm{N} / 22^{\circ} 53^{\prime} 05^{\prime \prime} \mathrm{E}$ & 276 & - & $4 \hat{\jmath}, 5$ 우 \\
\hline Băcaia & $46^{\circ} 00^{\prime} 59^{\prime \prime} \mathrm{N} / 23^{\circ} 10^{\prime} 27^{\prime \prime} \mathrm{E}$ & 370 & - & $2 \jmath^{\pi}, 6$ 우우 \\
\hline Conop & $46^{\circ} 07^{\prime} 34^{\prime \prime} \mathrm{N} / 21^{\circ} 53^{\prime} 13^{\prime \prime} \mathrm{E}$ & 180 & - & $2 \hat{\partial}$ \\
\hline Cladoviţa & 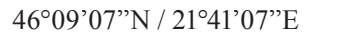 & 200 & - & 6 우우 \\
\hline Balşa & $46^{\circ} 03 ’ 12^{\prime \prime} \mathrm{N} / 23^{\circ} 04^{\prime} 32^{\prime \prime} \mathrm{E}$ & 360 & - & $2 \pi ⿱ 一,, 690$ \\
\hline Geoagiu & $46^{\circ} 01^{\prime} 12^{\prime \prime} \mathrm{N} / 23^{\circ} 07^{\prime} 17^{\prime \prime} \mathrm{E}$ & 330 & - & 9 ठึ, 11 우 \\
\hline Valea Țălnei & $46^{\circ} 10^{\prime} 47^{\prime \prime} \mathrm{N} / 22^{\circ} 29^{\prime} 16^{\prime \prime} \mathrm{E}$ & 420 & - & $3 \hat{\jmath}$ \\
\hline Văleni & $46^{\circ} 05^{\prime} 22^{\prime \prime} \mathrm{N} / 23^{\circ} 25^{\prime} 37^{\prime \prime} \mathrm{E}$ & 340 & - & 1 \\
\hline Vâltorii & $46^{\circ} 08^{\prime} 29^{\prime \prime} \mathrm{N} / 23^{\circ} 12^{\prime} 54^{\prime \prime} \mathrm{E}$ & 690 & - & $2 \hat{\jmath}, 1$ ㅇ \\
\hline \multicolumn{5}{|c|}{ Crişul Alb hydrographic basin } \\
\hline Valea Stoiaca & $46^{\circ} 12^{\prime} 59^{\prime \prime} \mathrm{N} / 21^{\circ} 44^{\prime} 23^{\prime \prime} \mathrm{E}$ & 250 & - & $1 \delta$ \\
\hline Nadăş & $46^{\circ} 12^{\prime} 38^{\prime \prime} \mathrm{N} / 21^{\circ} 55^{\prime} 20^{\prime \prime} \mathrm{E}$ & 240 & - & $4 \widehat{\jmath} \widehat{\jmath}, 10$ 우 \\
\hline Valea Megheş & $46^{\circ} 28^{\prime} 14^{\prime \prime} \mathrm{N} / 22^{\circ} 15^{\prime} 55^{\prime \prime} \mathrm{E}$ & 370 & $1 \hat{\sigma}, 1 q$ & - \\
\hline Țebea & $46^{\circ} 08^{\prime} 46^{\prime \prime} \mathrm{N} / 22^{\circ} 42^{\prime} 08^{\prime \prime} \mathrm{E}$ & 350 & - & $3 \delta^{\lambda}, 4$ 우 \\
\hline Valea Birtinului & $46^{\circ} 09^{\prime} 40^{\prime \prime} \mathrm{N} / 22^{\circ} 38^{\prime} 40^{\prime \prime} \mathrm{E}$ & 290 & - & 3 우우 \\
\hline Derjana & $46^{\circ} 13^{\prime} 49^{\prime \prime} \mathrm{N} / 22^{\circ} 42^{\prime} 32^{\prime \prime} \mathrm{E}$ & 280 & - & 2 우우 \\
\hline Rănuşa & $46^{\circ} 26^{\prime} 21^{\prime \prime} \mathrm{N} / 22^{\circ} 16^{\prime} 02^{\prime \prime} \mathrm{E}$ & 250 & 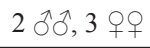 & - \\
\hline Valea Prundu & $46^{\circ} 12^{\prime} 37^{\prime \prime} \mathrm{N} / 22^{\circ} 06^{\prime} 16^{\prime \prime} \mathrm{E}$ & 340 & - & 1 q \\
\hline Aciua & $46^{\circ} 21^{\prime} 46^{\prime \prime} \mathrm{N} / 22^{\circ} 29^{\prime} 00^{\prime \prime} \mathrm{E}$ & 270 & - & $3 \hat{\jmath}, 4$ 우 \\
\hline \multicolumn{5}{|c|}{ Crişul Negru hydrographic basin } \\
\hline Valea Archişel & $46^{\circ} 30^{\prime} 22^{\prime \prime} \mathrm{N} / 22^{\circ} 07^{\prime} 53^{\prime \prime} \mathrm{E}$ & 390 & - & 3 우 \\
\hline Valea Zerzegului & $46^{\circ} 37^{\prime} 47^{\prime \prime} \mathrm{N} / 22^{\circ} 09^{\prime} 00^{\prime \prime} \mathrm{E}$ & 450 & $1 \hat{\delta}, 2$ 우 & - \\
\hline Valea Armanului & $46^{\circ} 38^{\prime} 43^{\prime \prime} \mathrm{N} / 22^{\circ} 13^{\prime} 38^{\prime \prime} \mathrm{E}$ & 300 & $1 \hat{\jmath}$ & - \\
\hline Sohodol & $46^{\circ} 50^{\prime} 41^{\prime \prime} \mathrm{N} / 22^{\circ} 22^{\prime} 21^{\prime \prime} \mathrm{E}$ & 350 & 1 ㅇ, 3 우우 & - \\
\hline Cuților & $46^{\circ} 51^{\prime} 14^{\prime \prime} \mathrm{N} / 22^{\circ} 25^{\prime} 17^{\prime \prime} \mathrm{E}$ & 525 & 2 우우 & - \\
\hline Tâlniciorii & $46^{\circ} 25^{\prime} 00^{\prime \prime} \mathrm{N} / 22^{\circ} 27^{\prime} 48^{\prime \prime} \mathrm{E}$ & 480 & $3 \widehat{\delta}, 6$ 우 & - \\
\hline Valea Rudăreasa & $46^{\circ} 28^{\prime} 10^{\prime \prime} \mathrm{N} / 22^{\circ} 31^{\prime} 00^{\prime \prime} \mathrm{E}$ & 360 & $2 \hat{\delta} \hat{\delta}, 2$ 우 & - \\
\hline Valea Sighiştel & $46^{\circ} 31^{\prime} 33^{\prime \prime} \mathrm{N} / 22^{\circ} 33^{\prime} 32^{\prime \prime} \mathrm{E}$ & 380 & 1 ㅇ & - \\
\hline Racăş & $46^{\circ} 51^{\prime} 51^{\prime \prime} \mathrm{N} / 22^{\circ} 18^{\prime} 42^{\prime \prime} \mathrm{E}$ & 220 & - & 1 ग, 2 우우 \\
\hline
\end{tabular}


Table 2 (continued)

\begin{tabular}{|c|c|c|c|c|}
\hline $\begin{array}{l}\text { Rivers } \\
\text { toponyms }\end{array}$ & Geographic coordinates & $\begin{array}{l}\text { 氖 } \\
\text { 导 } \\
\text { 弄 }\end{array}$ & 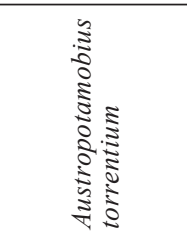 & 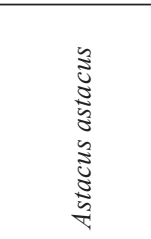 \\
\hline Crăiasa & $46^{\circ} 32^{\prime} 48^{\prime \prime} \mathrm{N} / 22^{\circ} 35^{\prime} 41^{\prime \prime} \mathrm{E}$ & 530 & 5 ふふર, 4 우 & - \\
\hline Boga & $46^{\circ} 36^{\prime} 31^{\prime \prime} \mathrm{N} / 22^{\circ} 39^{\prime} 33^{\prime \prime} \mathrm{E}$ & 560 & 1 우 & - \\
\hline Valea Finişului & $46^{\circ} 34^{\prime} 10^{\prime \prime} \mathrm{N} / 22^{\circ} 13^{\prime} 50^{\prime \prime} \mathrm{E}$ & 445 & 1 우 & - \\
\hline Valea Mare & $46^{\circ} 38^{\prime} 30^{\prime \prime} \mathrm{N} / 22^{\circ} 14^{\prime} 41^{\prime \prime} \mathrm{E}$ & 250 & - & 2 ふふ, 2 우우 \\
\hline Şoimuşurilor & $46^{\circ} 48^{\prime} 31^{\prime \prime} \mathrm{N} / 22^{\circ} 25^{\prime} 27^{\prime \prime} \mathrm{E}$ & 350 & $3 \widehat{\partial} \widehat{\partial}$ & - \\
\hline \multicolumn{5}{|c|}{ Crişul Repede hydrographic basin } \\
\hline Luncşoru & $47^{\circ} 06^{\prime} 27^{\prime \prime} \mathrm{N} / 22^{\circ} 36^{\prime} 44^{\prime \prime} \mathrm{E}$ & 380 & 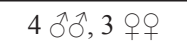 & - \\
\hline Valea Mare & $47^{\circ} 07^{\prime} 39^{\prime \prime} \mathrm{N} / 22^{\circ} 36^{\prime} 39^{\prime \prime} \mathrm{E}$ & 310 & 26 ふోَ, 28 우 & - \\
\hline Poicu & $46^{\circ} 59^{\prime} 05^{\prime \prime} \mathrm{N} / 22^{\circ} 55^{\prime} 12^{\prime \prime} \mathrm{E}$ & 625 & 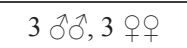 & - \\
\hline Valea Boului & $47^{\circ} 01^{\prime} 52^{\prime \prime} \mathrm{N} / 22^{\circ} 51^{\prime} 27^{\prime \prime} \mathrm{E}$ & 410 & - & 1 우 \\
\hline Barcău & $47^{\circ} 03^{\prime} 01^{\prime \prime} \mathrm{N} / 22^{\circ} 44^{\prime} 50^{\prime \prime} \mathrm{E}$ & 425 & - & 3 우우 \\
\hline Valea Peştilor & $47^{\circ} 06^{\prime} 11^{\prime \prime} \mathrm{N} / 22^{\circ} 39^{\prime} 39^{\prime \prime} \mathrm{E}$ & 315 & - & $3 \hat{\partial}^{\lambda}, 1$ 우 \\
\hline Valea Ieduţului & $46^{\circ} 41^{\prime} 28^{\prime \prime} \mathrm{N} / 22^{\circ} 35^{\prime} 11^{\prime \prime} \mathrm{E}$ & 800 & 4 ふึ่, 5 우 & - \\
\hline Valea Runcu & $46^{\circ} 45^{\prime} 40^{\prime \prime} \mathrm{N} / 22^{\circ} 34^{\prime} 03^{\prime \prime} \mathrm{E}$ & 630 & $2 \hat{\jmath}, 1$ ㅇ & - \\
\hline Răchiteasca & $47^{\circ} 03^{\prime} 30^{\prime \prime} \mathrm{N} / 22^{\circ} 31^{\prime} 51^{\prime \prime} \mathrm{E}$ & 290 & 1 ई, 3 우우 & - \\
\hline Valea Gârbului & $46^{\circ} 56^{\prime} 01^{\prime \prime} \mathrm{N} / 22^{\circ} 52^{\prime} 21^{\prime \prime} \mathrm{E}$ & 460 & 1 우 & - \\
\hline Valea Vişagului & $46^{\circ} 51^{\prime} 51^{\prime \prime} \mathrm{N} / 22^{\circ} 48^{\prime} 52^{\prime \prime} \mathrm{E}$ & 590 & 1 우 & - \\
\hline Valea Bisericii & $46^{\circ} 50^{\prime} 08^{\prime \prime} \mathrm{N} / 22^{\circ} 40^{\prime} 13^{\prime \prime} \mathrm{E}$ & 495 & $2 \widehat{\jmath}, 2$ +q & - \\
\hline Damiş & $46^{\circ} 52^{\prime} 00^{\prime \prime} \mathrm{N} / 22^{\circ} 32^{\prime} 12^{\prime \prime} \mathrm{E}$ & 700 & 2 ふふ人, 4 우 & - \\
\hline Valea Mierei & $46^{\circ} 58^{\prime} 05^{\prime \prime} \mathrm{N} / 22^{\circ} 24^{\prime} 30^{\prime \prime} \mathrm{E}$ & 455 & - & 10,1 q \\
\hline \multicolumn{5}{|c|}{ Someş hydrographic basin } \\
\hline Vicleanul Mare & $47^{\circ} 40^{\prime} 29^{\prime \prime} \mathrm{N} / 23^{\circ} 36^{\prime} 26^{\prime \prime} \mathrm{E}$ & 290 & - & $3 \hat{\jmath}, 1$ q \\
\hline Valea Usturoiului & $47^{\circ} 41^{\prime} 04^{\prime \prime} \mathrm{N} / 23^{\circ} 34^{\prime} 24^{\prime \prime} \mathrm{E}$ & 320 & - & 3 우 \\
\hline Valea Mare & $47^{\circ} 43^{\prime} 06^{\prime \prime} \mathrm{N} / 23^{\circ} 25^{\prime} 05^{\prime \prime} \mathrm{E}$ & 280 & - & $10^{\pi}$ \\
\hline Agrij & $47^{\circ} 01^{\prime} 10^{\prime \prime} \mathrm{N} / 22^{\circ} 58^{\prime} 51^{\prime \prime} \mathrm{E}$ & 425 & - & 1 సิ, 2 우우 \\
\hline Gârbău & $46^{\circ} 43^{\prime} 54^{\prime \prime} \mathrm{N} / 23^{\circ} 31^{\prime} 52^{\prime \prime} \mathrm{E}$ & 350 & - & $10^{\pi}$ \\
\hline Someşul Cald & $46^{\circ} 43^{\prime} 50^{\prime \prime} \mathrm{N} / 23^{\circ} 18^{\prime} 47^{\prime \prime} \mathrm{E}$ & 430 & - & 3 우우 \\
\hline Tetişu & $46^{\circ} 54^{\prime} 45^{\prime \prime} \mathrm{N} / 23^{\circ} 03^{\prime} 22^{\prime \prime} \mathrm{E}$ & 340 & - & $1 \sigma^{\pi}$ \\
\hline \multicolumn{5}{|c|}{ Tisa tributaries } \\
\hline Valea Holița & $48^{\circ} 05^{\prime} 07^{\prime \prime} \mathrm{N} / 23^{\circ} 13^{\prime} 03^{\prime \prime} \mathrm{E}$ & 210 & - & 3 우우 \\
\hline Valea Mare & $48^{\circ} 00^{\prime} 46^{\prime \prime} \mathrm{N} / 23^{\circ} 18^{\prime} 21^{\prime \prime} \mathrm{E}$ & 200 & - & $1 \delta^{\Uparrow}$ \\
\hline Sărăsău & $47^{\circ} 56^{\prime} 53^{\prime \prime} \mathrm{N} / 23^{\circ} 47^{\prime} 52^{\prime \prime} \mathrm{E}$ & 300 & - & 5 ふえ, 7 우 \\
\hline Băleaşa & $47^{\circ} 38^{\prime} 14^{\prime \prime} \mathrm{N} / 24^{\circ} 20^{\prime} 59^{\prime \prime} \mathrm{E}$ & 540 & - & 1 ㅇ \\
\hline Valea Tejei & $47^{\circ} 48^{\prime} 03^{\prime \prime} \mathrm{N} / 23^{\circ} 53^{\prime} 29^{\prime \prime} \mathrm{E}$ & 440 & - & $1 \delta, 1 q$ \\
\hline
\end{tabular}


rivers. In the Hungarian lower course of the Tisa River, the presence of $O$. limosus has already been reported (Puky \& Schád, 2006; Puky, 2009). The worst threat, represented by Pacifastacus leniusculus, should be taken into consideration over the next decades. In Hungary, this species is reported in Rába River (Puky et al., 2005), and it is known to be more susceptible to colonization of the smaller tributaries, even brooks and springs (Holdich et al., 2006): this creates the possibility of contact with A. torrentium, and therefore transmission of the crayfish plague infection to their populations, considerably higher.

\section{ACKNOWLEDGEMENTS}

This study was funded by CNCSIS - Exploratory research projects PCE-4 grant no 1458/2008 "The stone crayfish (Austropotamobius torrentium), distribution in Romanian habitats, ecology and genetics of populations". I want to thank the Romanian Academy, especially The Commission for Natural Protected Monuments, for approving this study, as well as the administrators of the natural protected areas for permission to work in the field. Also, many thanks to Zoltan Kovacs and Zoltan Mate for their help in the Plopiş Mountains investigations. Last, but not least, I want to thank to the volunteer students of the Faculty of Chemistry, Biology and Geography from the West University of Timişoara for participation in the field activities. The anonymous reviewers provided useful suggestions on a previous version of the manuscript.

\section{DISTRIBUȚIA ȘI PREFERINȚELE ECOLOGICE ALE RACULUI-DE-PONOARE AUSTROPOTAMOBIUS TORRENTIUM (SCHRANK, 1803) (DECAPODA: ASTACIDAE) ÎN NORD-VESTUL ROMÂNIEI}

\section{REZUMAT}

În această lucrare sunt prezentate date referitoare la distribuţia geografică şi preferinţele ecologice ale speciei Austropotamobius torrentium în urma investigării a 104 stații de prelevare din zona de vest şi de nord-vest a României. Exemplarele au fost capturate activ, căutând în albia râului, după care au fost eliberate. A fost realizată o hartă detaliată de distribuţie, specia fiind găsită la 23 din stațiile de prelevare făcând parte din bazinele râurilor Crişul Alb, Crişul Negru şi Crişul Repede. Aria actuală de răspândire a speciei ocupă centrul şi estul Munţilor Codru Moma, vestul Munţilor Bihor, estul Munţilor Pădurea Craiului, centrul şi nordul Munţilor Vlădeasa, şi sudul şi centrul Munţilor Plopiş. Cea mai nordică populaţie din România este reprezentată de râul Valea Mare din Munţii Plopiş. În general, specia ocupă cursuri de apă cu un substrat format din pietre şi pietriş. Combinaţia de vegetaţie ripariană preferată este Alnus sp. şi Salix sp. în etajul pădurilor de foioase. La un număr important de pâraie investigate, 37 din numărul total, a fost găsită specia Astacus astacus, datele arătând că nu există populaţii mixte. Nicio specie invazivă nu a fost găsită în timpul acestui studiu.

\section{LITERATURE CITED}

BĂCESCU, M. C., 1967 - Crustacea, Decapoda. In: Fauna Republicii Socialiste România. Edit. Academiei Republicii Socialiste România, Bucureşti, 4: 209-219. (in Romanian)

ENTZ, G., 1912 - Über die Flußkrebse Ungarns. Mathematischen Naturwissenschaftlichen Berichte aus Ungarn, 30 (2): 67-125.

FÜREDER, L., F. GHERARDI, C. SOUTY-GROSSET, 2010 - Austropotamobius torrentium. In: IUCN 2011. IUCN Red List of Threatened Species. Version 2011.1. <www.iucnredlist.org>. Downloaded on 24 October 2011.

FÜREDER, L., B. OBERKOFLER, R. HANEL, J. LEITER, B. THALER, 2003 - The freshwater crayfish Austropotamobius pallipes in South Tyrol: heritage species and bioindicator. Bulletin Français de la Pêche et de la Pisciculture, 370-371: 79-95.

GHERARDI, F., D. M. HOLDICH, 1999 - Crayfish in Europe as alien species. How to make the best of a bad situation. Crustacean Issues 11, A. A. Balkema, Rotterdam, Brookfield, 299 pp.

HOLDICH, D. M., P. HAFFNER, P. Y. NOËL, 2006 - Species files. In: C. Souty-Grosset, D. M. Holdich, P. Y. Noël, J. D. Reynolds, P. Haffner (eds), Atlas of Crayfish in Europe. Muséum National d'Histoire Naturelle, Paris, (Patrimoines Naturels, 64): 49-129.

HOLDICH, D. M., J. D. REYNOLDS, C. SOUTY-GROSSET, P. J. SIBLEY, 2009 - A review of the ever increasing threat to European crayfish from non-indigenous crayfish species. Knowledge and Management of Aquatic Ecosystems, 394-395, 11: 1-46. 
LOWERY, R., J. HOGGER, 1986 - The effect of river engineering and disease on a population of Austropotamobius pallipes in the River Lea, UK. Freshwater Crayfish, 6: 94-97.

LYONS, R., M. KELLY-QUINN, 2003 - An investigation into the disappearance of Austropotamobius pallipes (Lereboullet) populations in the headwaters of the Nore River, Ireland and the correlation to water quality. Bulletin Français de la Pêche et de la Pisciculture, 370-371: $139-150$.

MACHINO, Y., L. FÜREDER, 2005 - How to find a stone crayfish Austropotamobius torrentium (Schrank, 1803): a biogeography study in Europe. Bulletin Français de la Pêche et de la Pisciculture, 376-377: 507-517.

MAGUIRE, I., A. LUCIĆ, M. SCHLETTERER, D. SINT, R. ERBEN, L. FÜREDER, 2010 - Fecundity of Indigenous Stone Crayfish, Austropotamobius torrentium, Populations: Implications for Crayfish Conservation in Austria and Croatia. Freshwater Crayfish, 17: 103-107.

PÂRVULESCU, L., 2007 - Austropotamobius torrentium. Pp. 47-49. In: I. Combroux, E. Thiry, T. Ţoia (eds), Caiet de habitate şi specii - Fişe pilot. Edit. Balcanic, Timişoara. (in Romanian)

PÂRVULESCU, L., 2010 - Crayfish field guide of Romania. Edit. Bioflux, Cluj-Napoca: 28 p.

PÂRVULESCU, L., I. PETRESCU, 2010 - The distribution of stone crayfish Austropotamobius torrentium (Schrank, 1803) (Crustacea: Decapoda: Astacidae) in the south-west Romanian mountain and sub-mountain area. Travaux du Muséum National d'Histoire Naturelle "Grigore Antipa", 53: 103-113.

PÂRVULESCU, L., O. PACIOGLU, C. HAMCHEVICI, 2011 - The assessment of the habitat and water quality requirements of the stone crayfish (Austropotamobius torrentium) and noble crayfish (Astacus astacus) species in the rivers from the Anina Mountains (SW Romania). Knowledge and Management of Aquatic Ecosystems, 401, 03: 1-12.

PÂRVULESCU, L., C. PALOŞ, P. MOLNAR, 2009 - First record of spiny-cheek crayfish Orconectes limosus (Rafinesque, 1817) (Crustacea: Decapoda: Cambaridae). North-Western Journal of Zoology, 5 (2): 424-428.

PÂRVUlESCU, L., A. SCHRIMPF, E. KOZUBÍKOVÁ, S. C. RESINO, T. VRÅlSTAD, A. PETRUSEK, R. SCHULZ, 2012 - Invasive crayfish and crayfish plague on the move: first detection of the plague agent Aphanomyces astaci in the Romanian Danube. Diseases of Aquatic Organisms, 98: 85-94.

PETRESCU, I., A. M. PETRESCU, 2010 - The catalogue of the freshwater crayfish (Crustacea: Decapoda: Astacidae) from Romania preserved in "Grigore Antipa" National Museum of Natural History of Bucharest. Travaux du Muséum National d'Histoire Naturelle "Grigore Antipa", 53: 115-123.

PETRUSEK, A., L. FILIPOVA, Z. ĎURIS, I. HORKA, P. KOZAK, T. POLICAR, M. ŠTAMBERGOVA, Z. KUCERA, 2006 - Distribution of the invasive spiny-cheek crayfish (Orconectes limosus) in the Czech Republic. Past and present. Bulletin Français de la Pêche et de la Pisciculture, 380-381: 903-917.

PUKY, M., 2009 - Confirmation of the presence of the spiny-cheek crayfish Orconectes limosus (Rafinesque, 1817) (Crustacea: Decapoda: Cambaridae) in Slovakia. North-Western Journal of Zoology, 5: 214-217.

PUKY, M., P. SCHAD, 2006 - Orconectes limosus colonises new areas fast along the Danube in Hungary. Bulletin Français de la Pêche et de la Pisciculture, 380-381: 919-926.

PUKY, M., J. D. REYNOLDS, P. SCHÁD, 2005 - Native and alien decapoda species in Hungary: distribution, status, conservation importance. Bulletin Français de la Pêche et de la Pisciculture, 376-377: 553-568.

SCRIBAN, I. A., 1908 - Note sur les Ecrevisses de Roumanie. Annales Scientifiques de l'Université de Jassy, 5: 80-83.

STREISSL, F., W. HÖDL, 2002 - Growth, morphometrics, size at maturity, sexual dimorphism and condition index of Austropotamobius torrentium Schrank. Hydrobiologia, 477: 201-208.

SVOBODOVÁ, J., M. ŠTAMBERGOVÁ, P. VLACH, J. PICEK, K. DOUDA, M. BERÁNKOVÁ, 2008 - The impact of the water quality on the crayfish population in the Czech Republic, comparison with legislation of the Czech Republic. VTEI, 50: 1-5.

VLACH, P., D. FISCHER, L. HULEC, 2009 - Microhabitat preferences of the stone crayfish Austropotamobius torrentium (Schrank, 1803). Knowledge and Management of Aquatic Ecosystems, 394-395, 15: 1-13.

*** EUROPEAN COMMUNITIES, 1992 - Council Directive (92/43/EEC) on the conservation of natural habitats and of wild fauna and flora, The Council of European Communities. 
Received: October 27, 2011 Accepted: July 23, 2012
West University of Timişoara

Faculty of Chemistry, Biology, Geography

Department of Biology-Chemistry

Pestalozzi 16A St., 300115 Timișoara, Romania

e-mail: parvulescubio@cbg.uvt.ro 